\title{
Myocardial MiR-30 downregulation triggered by doxorubicin drives alterations in $\beta$-adrenergic signaling and enhances apoptosis
}

\author{
L Roca-Alonso*,1 , L Castellano ${ }^{*, 1}$, A Mills ${ }^{2}$, AF Dabrowska ${ }^{1}$, MB Sikkel $^{2}$, L Pellegrino ${ }^{1}$, J Jacob ${ }^{1}$, AE Frampton ${ }^{1,3}$, J Krell ${ }^{1}$, RC Coombes ${ }^{1}$, \\ SE Harding ${ }^{2}$, AR Lyon ${ }^{2,4,5}$ and J Stebbing ${ }^{1,5}$
}

The use of anthracyclines such as doxorubicin (DOX) has improved outcome in cancer patients, yet associated risks of cardiomyopathy have limited their clinical application. DOX-associated cardiotoxicity is frequently irreversible and typically progresses to heart failure (HF) but our understanding of molecular mechanisms underlying this and essential for development of cardioprotective strategies remains largely obscure. As microRNAs (miRNAs) have been shown to play potent regulatory roles in both cardiovascular disease and cancer, we investigated miRNA changes in DOX-induced HF and the alteration of cellular processes downstream. Myocardial miRNA profiling was performed after DOX-induced injury, either via acute application to isolated cardiomyocytes or via chronic exposure in vivo, and compared with miRNA profiles from remodeled hearts following myocardial infarction. The miR-30 family was downregulated in all three models. We describe here that miR-30 act regulating the $\beta$-adrenergic pathway, where preferential $\beta 1$ - and $\beta 2$-adrenoceptor ( $\beta 1 \mathrm{AR}$ and $\beta 2 \mathrm{AR}$ ) direct inhibition is combined with Gi $\alpha-2$ targeting for fine-tuning. Importantly, we show that miR-30 also target the pro-apoptotic gene BNIP3L/NIX. In aggregate, we demonstrate that high miR-30 levels are protective against DOX toxicity and correlate this in turn with lower reactive oxygen species generation. In addition, we identify GATA-6 as a mediator of DOX-associated reductions in miR-30 expression. In conclusion, we describe that DOX causes acute and sustained miR-30 downregulation in cardiomyocytes via GATA-6. miR-30 overexpression protects cardiac cells from DOX-induced apoptosis, and its maintenance represents a potential cardioprotective and anti-tumorigenic strategy for anthracyclines.

Cell Death and Disease (2015) 6, e1754; doi:10.1038/cddis.2015.89; published online 7 May 2015

Doxorubicin (DOX) is the most used anthracycline in the oncology clinic, delivering anti-tumor activity against numerous types of malignancy. ${ }^{1,2}$ The exact mechanisms accounting for the anti-cancer action(s) of anthracyclines remain poorly understood, although a variety of effects have been proposed, most notably inhibition of topoisomerase II (Top2). ${ }^{2}$ Patients receiving DOX treatment often experience adverse effects, the most serious being dose-dependent cardiotoxicity that can lead to heart failure (HF). Efficient prevention and early diagnosis of DOX-induced cardiotoxicity is essential, as once patients develop advanced HF, their prognosis is poor. ${ }^{3}$

Mechanistically, reactive oxygen species (ROS) and oxidative stress have been linked to DOX-induced cardiotoxicity ${ }^{4,5}$ and could explain the relative tissue specificity of the adverse effects of DOX as the heart is rich in mitochondria, metabolically active and has relatively low antioxidant-producing enzymes. ${ }^{6}$ Others have highlighted the cardioprotective effects of cardiomyocyte-specific Top2 $\beta$ deletion, which suggest this enzyme could mediate the pathological side effects of DOX. ${ }^{7}$

Alterations in myocardial responses to catecholamines are well described in HF. Physiologically, $\beta$-adrenoceptor ( $\beta A R$ ) activation by catecholamines results in protein $G\left(G_{s}\right)$ dependent activation of adenylyl cyclase (AC), enhancing cyclic adenosine monophosphate (cAMP) production from ATP. CAMP activates protein kinase A (PKA), which is responsible for phosphorylating several proteins involved in $\mathrm{Ca}^{2+}$ metabolism and contraction. Although $\beta_{1} \mathrm{AR}$ is only able to interact with $G_{s}, \beta_{2} A R$ can bind both to $G_{s}$ and to the inhibitory $G_{i}$ protein; $\beta_{2} A R-G_{i}$ activation reduces contraction via both cAMP-dependent and -independent mechanisms. Chronic sympathetic activation is detrimental for the heart and sustained overexpression of $\beta_{1} \mathrm{AR}$ triggers myocyte apoptosis and hypertrophy leading to $\mathrm{HF}^{8}$ Sustained $\beta$-adrenergic stimulation leads to reduced expression and/or compensatory

\footnotetext{
${ }^{1}$ Division of Oncology, Department of Surgery and Cancer, 1st Floor, Imperial Centre for Translational and Experimental Medicine (ICTEM), Imperial College, Hammersmith Hospital, Du Cane Road, London W12 ONN, UK; ${ }^{2}$ National Heart and Lung Institute, Imperial College, 4th Floor, ICTEM, Hammersmith Hospital, Du Cane Road, London W12 0NN, UK; ${ }^{3}$ Hepato-Pancreato-Biliary Surgical Unit, Department of Surgery and Cancer, Imperial College, Hammersmith Hospital, Du Cane Road, London W12 0NN, UK and ${ }^{4}$ NIHR Cardiovascular Biomedical Research Unit, Royal Brompton Hospital, Imperial college, London SW3 6NP, UK

${ }^{*}$ Corresponding author: L Roca-Alonso or L Castellano, Division of Oncology, Department of Surgery and Cancer, 1st Floor, Imperial Centre for Translational and Experimental Medicine (ICTEM), Imperial College, Hammersmith Hospital, Du Cane Road, London W12 ONN, UK. Tel: +44 2075942823; Fax: +44 203 3111433; E-mail: I.roca.alonso@gmail.com or I.castellano@imperial.ac.uk

${ }^{5}$ These authors contributed equally to this work.

Abbreviations: $\beta$ AR, $\beta$-adrenergic receptor; AC, Adenylyl cyclase; AMC, Age-matched control; AR, Adrenoceptor; ARVCM, Adult rat ventricular cardiomyocytes; Ca ${ }^{2+}$, Calcium; cAMP, 3',5'-Cyclic adenosine monophosphate; DOX, Doxorubicin; Gi, Guanine-coupled inhibitory protein; Gs, Guanine-coupled stimulatory protein; GTP, Guanine triphosphate; HF, Heart failure; LAD, Left anterior descendent; LNA, Locked nucleic acid; MI, Myocardial Infarction; miRNA, microRNA; mRNA, Messenger RNA; NC, Negative control; PI3-K, Phosphoinositide 3-kinase; PKA, Protein kinase A; ROS, Reactive oxygen species; RT-qPCR, Reverse transcription-quantitative PCR Received 18.8.14; revised 14.12.14; accepted 12.1.15; Edited by M Agostini
} 
desensitization of $\beta A R s$ to catecholamines, both features of the functionally impaired heart. ${ }^{9}$ Increased expression of $G_{i}-$ particularly of the $a-2$ subunit $\left(G_{i a-2}\right)$-is also observed in HF, where enhanced $\beta_{2} A R-G_{i}$ coupling triggers diminished basal contractility following receptor activation. However, the mechanisms underpinning Gi upregulation in chronic HF have remained unknown. ${ }^{10,11} \beta$-Adrenergic antagonists ( $\beta$-blockers) constitute a critical clinical advance in the treatment of HF, being able to improve cardiac output. ${ }^{12,13}$

Contributing to the developing molecular picture of multiple diseases, microRNAs (miRNAs) are a class of short ( 22 nucleotides, nt) non-coding RNAs that play influential roles in the post-transcriptional 'fine-tuning' of gene expression. ${ }^{14}$ miRNAs are often coded in clusters and transcribed as single transcriptional units, each forming a primary miRNA, which is then processed and gives rise to multiple mature miRNAs. ${ }^{15,16}$ The miRNA classification into families groups together those miRNAs that share the same 'seed region', which is the sequence necessary for target messenger RNA (mRNA) recognition and is usually located between nucleotides 2 and $8 .{ }^{17}$ miRNAs are implicated in heart development and function ${ }^{18-20}$ and specific miRNA signatures have been associated with certain cardiac pathologies. ${ }^{21-24}$ Nonetheless, few studies have validated their functional impact. Although recent evidence describes the dysregulation of miRNAs across the heart upon DOX treatment, ${ }^{25,26}$ a cardiomyocyte-specific miRNA signature of DOX-triggered alterations, or their consequences, is currently lacking.

We identified the DOX-induced cardiomyocyte miRNA alterations in various models of cardiac toxicity. We then proceeded to uncover the regulation of pathways implicated in heart disease by the miR-30 family (miR-30), which we found to be downregulated by DOX. This miRNA family is formed by four clusters, located in different genomic regions and organized in the following way in both rat and human: miR-30a; miR-30c-2; miR-30b and miR-30d; miR-30e and miR-30c-1 (http://www.mirbase.org). We report that miR-30 fine-tunes the $\beta$-adrenergic pathway by targeting $\beta A R$ s and $G_{i a-2}$, while also inhibiting the expression of the pro-apoptotic gene BNIP3L/NIX. Overall, gene expression regulation by miR-30 seems to be protective against the toxic effects of DOX in cardiac cells.

\section{Results}

DOX triggers miR-30 family downregulation in cardiomyocytes. To obtain the miRNA signature of cardiomyocytes affected by DOX treatment, we established both in vitro and in vivo models. These included two different DOX treatments: an acute in vitro administration to primary isolated adult rat ventricular cardiomyocytes (ARVCMs) and an in vivo model of DOX-induced HF (Supplementary Figures 2 and 3), created by serial injections of the drug as previously described. ${ }^{27}$ An in vivo model of myocardial infarction (MI) was generated by proximal left anterior descendent (LAD) coronary artery ligation leading to pathological hypertrophy at 4 weeks and HF by 16-20 weeks and was included as reference of severe cardiomyopathy. ${ }^{28} \mathrm{~A}$ total of 26 miRNAs had significantly altered expression by $\geq 1.5$-folds in DOX- treated cultured ARVCMs, 18 miRNAs in ARVCMs obtained from DOX-treated hearts and 40 miRNAs were dysregulated in ARVCM from infarcted hearts 4 weeks post MI. Seven miRNAs (miR-133b, miR-143, miR-210, miR-29c, miR-30d, miR-30e and miR-345-5p) were found to be downregulated in all three models (Figure 1). A subset of three overlapping hits (miR-30e, miR-210 and miR-29c) was chosen for validation by reverse transcription-quantitative PCR (RT-qPCR; Supplementary Figure 4). Moreover, sustained miRNA alteration during $\mathrm{HF}$ progression was confirmed by RT-qPCR at a later time of 16-20 weeks post MI stage (Supplementary Figure 5). We could also confirm aberrant expression of several miRNAs that had previously linked to heart disease in our models (Figure 1b, bold writing). ${ }^{21,22,25,29-35}$ Strikingly, three members of the miR-30 family (miR-30a, miR-30d and miR-30e) were downregulated in at least two models, which indicated potential biological relevance. miR-30e showed the greatest dysregulation across all three models of study (Figure 1c).

DOX induces $\beta_{1} A R, \beta_{2} A R, G_{i q-2}$ and BNIP3L/NIX expression through miR-30 downregulation. To further investigate the implication of miR-30 in DOX-induced myocardial damage, we used TargetScan software and retrieved a list of highly conserved predicted miR-30 target genes, based on seed pairing. Given that all family members share the same seed sequence, they are predicted to target the same genes. ${ }^{36}$ Pathway enrichment analysis of putative miR-30 targets using Database for annotation, visualization and integrated discovery (http://david.abcc.ncifcrf.gov/) revealed an implication of this target list in cardiovascular disease, being 3 out of the 10 most significantly enriched pathways related to cardiomyopathy (Figure $2 \mathrm{a}$ ). Among the predicted miR-30 targets, we focused on four genes, which were identified as part of an interacting network by Cytoscape (Supplementary Figure 7) and thus considered highconfidence predictions. Three of these genes are involved in the $\beta$-adrenergic pathway: $\beta_{1} A R, \beta_{2} A R$ and $G_{i a-2}$. The fourth target selected was the BCL2/adenovirus E1Binteracting protein 3-like (BNIP3L or NIX), an important apoptotic effector capable of mediating mitochondrial death and triggering myocardial disease $\mathrm{s}^{37-40}$ (Figure $2 \mathrm{~b}$ ).

All four targets were first validated by luciferase assays using vectors containing the respective target 3'UTR downstream of the luciferase reporter gene (see Methods). Mutated versions of the 3'UTR sequences with disrupted miR-30-binding sites were also assayed to confirm specific miRNA-3'UTR interaction and the non-target programed cell death protein 4 (PDCD4) was included as additional negative control (NC). As cellular platform, we used $\mathrm{H} 9 \mathrm{c} 2$ cardiac muscle cell line, which retains numerous features of mature cardiomyocytes and have been previously used to investigate DOX cardiotoxicity ${ }^{41,42}$ (Supplementary Figure 6). A significant reduction in luciferase activity was recorded for all four predicted targets when cotransfecting with the corresponding 3'UTR vector and the miR-30e mimic (pre-30e) as representative of the family, compared with pre-NC. Conversely, as expected, no changes were observed when co-transfected with the mutated 3'UTR versions and for PDCD4 (Figure 2c). Overall, we demonstrated direct regulation of these four genes by miR-30. 

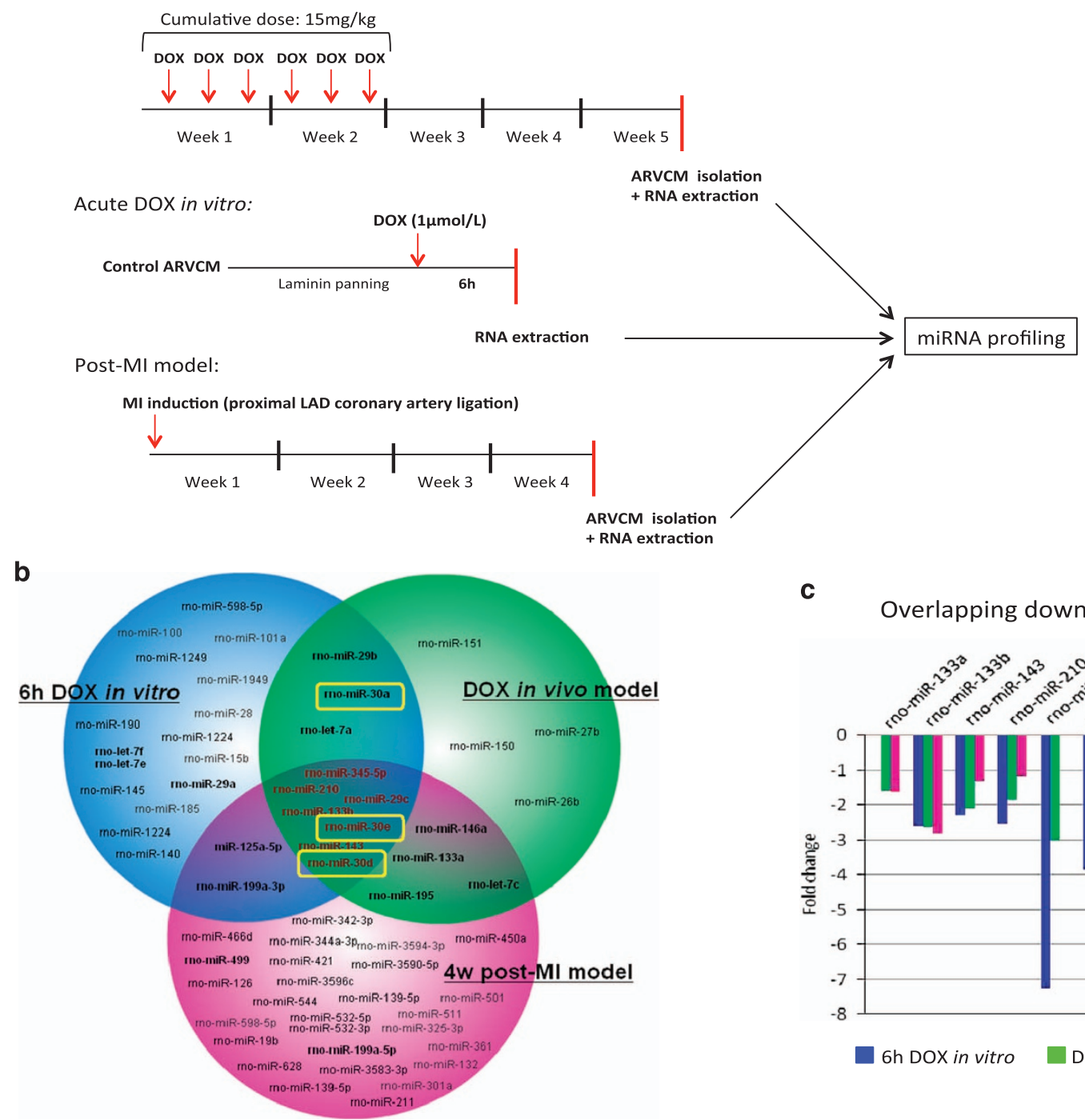

C

Overlapping down-regulated miRNAs

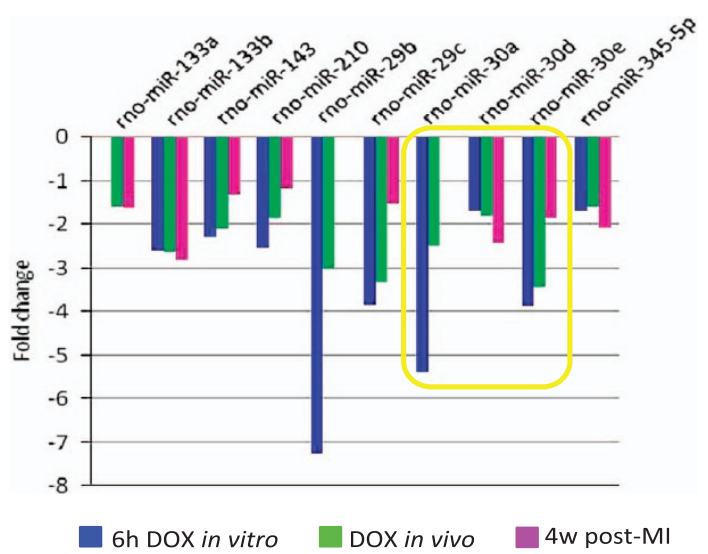

Figure 1 miRNA expression dysregulation occurs upon acute and sustained DOX treatment. (a) Experimental design of the models of study included in the miRNA profiling. DOX-induced HF in vivo model was generated by six i.p. DOX injections $(2.5 \mathrm{mg} / \mathrm{kg})$ spread over a fortnight. Ml was induced by proximal LAD coronary artery ligation. For acute DOX treatment, cultured viable ARVCM were treated with $1 \mu \mathrm{mol} / \mathrm{l}$ for $6 \mathrm{~h}$. For all three models, RNA was isolated specifically from viable ARVCM. (b) Venn diagram showing significantly altered miRNA expression ( $>1.5$-folds, $P<0.05$ ) detected for the profiled models of cardiac injury ( $n=3$ per group). The three downregulated members of the miR-30 family are highlighted in yellow. Several other miRNAs have previously been linked to heart disease when dysregulated in the same direction as we observed and appear in bold writing. (c) miRNAs that presented reduced expression levels in at least two experimental models. rno, rattus norvegicus.

We next overexpressed miR-30e in H9c2 cardiac cultures and observed a decrease in target mRNA levels of the selected targets (Figure $3 a$ ). We quantified protein dysregulation of BNIP3L and $G_{i a-2}$ by western blotting (Figure $3 b$ ) but this was not possible for $\beta_{1} A R$ and $\beta_{2} A R$ as there were no specific antibodies commercially available. ${ }^{43,44}$ To partly overcome this issue, accumulation of CAMP was used as an indicator of $\beta A R$ signaling, which validated miR-30 targeting (Figure 4a). Additional confirmation of miR-30-dependent regulation of target genes was achieved by miR-30 inhibition using an in-house sponge vector designed inhibit the entire miR-30 family ${ }^{45}$ (Figure $3 \mathrm{c}$ ). In contrast to the results obtained upon miR-30e overexpression, increased target mRNA and protein levels were recorded (Figures $3 d$ and e). These data were replicated independently using a commercial locked nucleic acid (LNA) construct for whole miR-30 family inhibition (Supplementary Figures 8 and 9). Remarkably, DOX treatment of H9c2 cardiac cells triggered an increase in miR-30 target mRNA levels, which could be fully reversed by simultaneous overexpression of miR-30 (Figure 3f). This indicates that mechanistically DOX is able to induce miR-30 target expression through miR-30 downregulation. Interestingly, miR-30 target upregulation also occurred in vivo upon DOX treatment, which is consistent with the demonstrated miR-30 downregulation in this disease model (Figure $3 \mathrm{~g}$ ). Together, these data suggest that $\beta_{1} A R, \beta_{2} A R, G_{i a-2}$ and BNIP3L/NIX are novel miR-30 targets and that the downregulation of miR-30 by DOX is responsible for the increase in target gene expression. 
miR-30 expression attenuates the contractile response of cardiomyocytes to $\beta$ AR stimulation. miRNAs can modulate the expression of hundreds of mRNAs and is the global regulatory effect on targets with opposing functions that will ultimately influence cellular phenotypes. After validating three members of the $\beta$-adrenergic pathway $\left(\beta_{1} A R, \beta_{2} A R\right.$ and $\left.G_{i a-2}\right)$ as miR-30 targets, we evaluated the net effects of miR-30 dysregulation on this pathway. As mentioned, cAMP is a

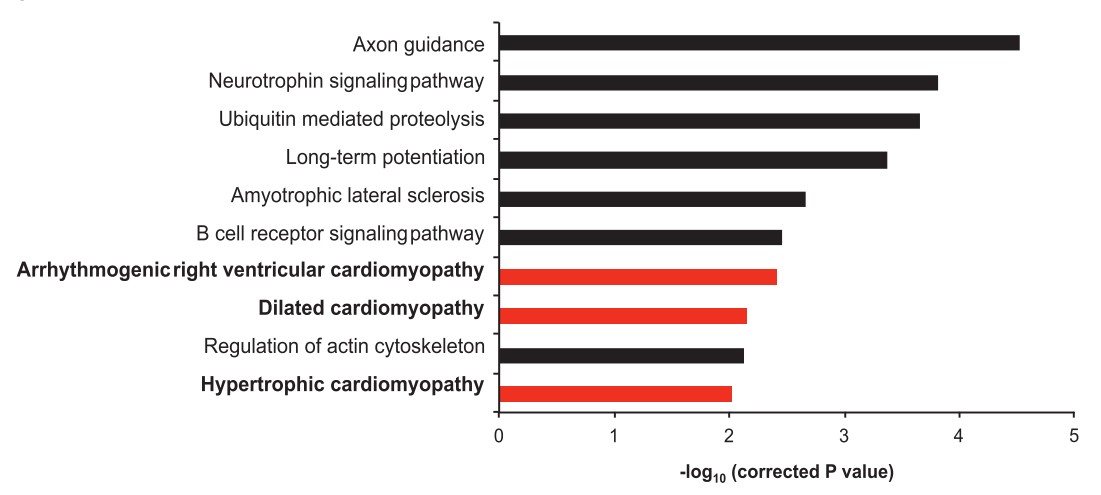

b

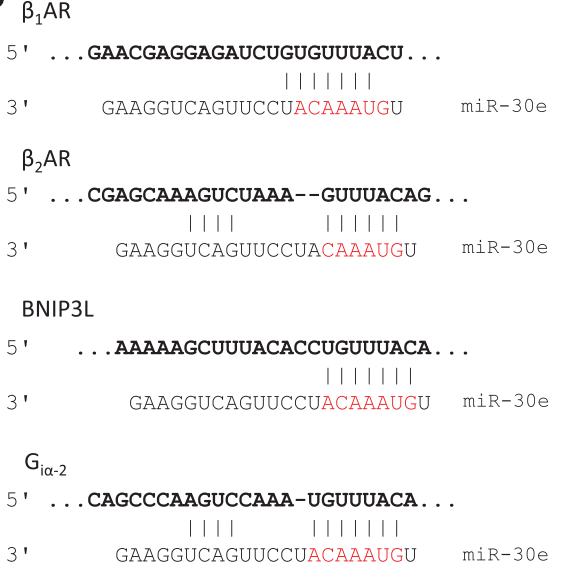

c
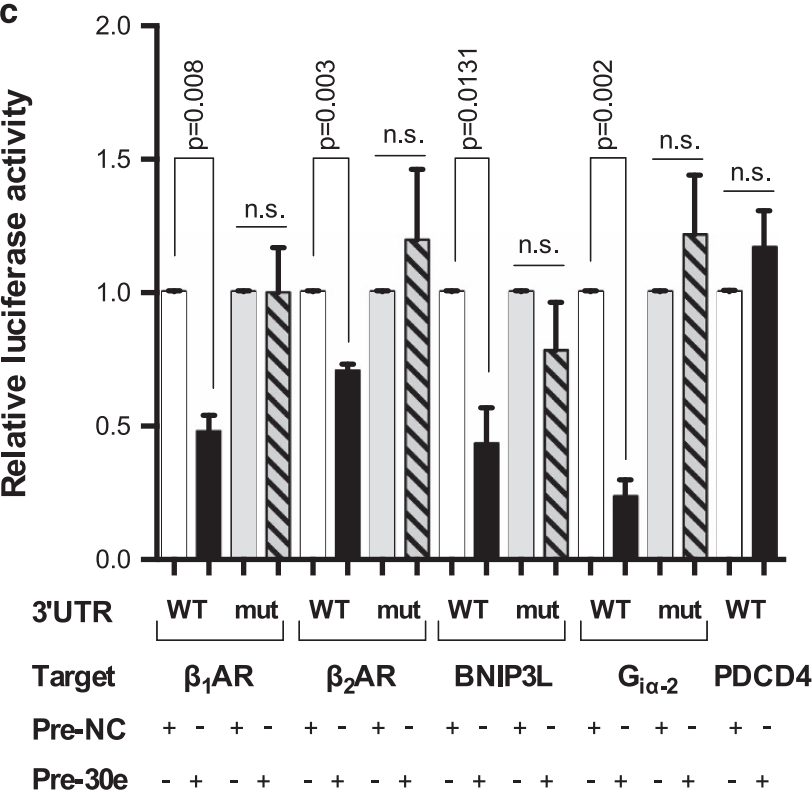

Figure 2 miR-30 target prediction and validation of direct $3^{\prime} U T R$ regulation by $3^{\prime} U T R$ luciferase assay. (a) Pathway enrichment analysis of the predicted miR-30 targets by TargetScan; performed using Database for annotation, visualization and integrated discovery. Three types of cardiomyopathy are part of the top 10 most significantly enriched pathways. (b) Representation of miR30e-mRNA annealing for four of the target genes predicted by TargetScan $\left(\beta_{1} A R, \beta_{2} A R, B N I P 3 L\right.$ and $\left.G_{i \alpha-2}\right)$. miRNA seed sequence is highlighted in red. (c) Relative luciferase activity measured on H9c2 cultures, after $24 \mathrm{~h}$ of co-transfection with either pre-30e or pre-NC (100 nmol/) and the correspondent (wild type (WT) or mutant (mut)) $3^{\prime} U T R$ luciferase reporter vector for each of the potential targets. The non-target programed cell death protein 4 (PDCD4) was included as negative control. Averaged ( \pm S.E.M.) values are the result of at least three independent transfections (ns, not significant; $t$-test)

Figure 3 Experimental confirmation of $\beta 1 \mathrm{AR}, \beta 2 \mathrm{AR}$, BNIP3L and Gi $\alpha-2$ as miR-30 targets. (a) RT-qPCR shows relative target expression levels when overexpressing miR-30. All values are normalized to U6 levels and presented as ratios to the pre-NC-transfected controls $(20 \mathrm{nmol} / \mathrm{l})$. Three biological replicates were performed on H9c2 cultures. (b) Relative protein levels of BNIP3L and $\mathrm{G}_{\mathrm{j}} \alpha_{-2}$ were measured by western blot; band densitometry was quantified by ImageJ and normalized to tubulin. (c) Schematic view of the sponge vector designed for miR-30 family inhibition (pEGFP-sp30), using the pEGFP-C1 plasmid as backbone. EGFP expression in transfected cultures was detected by microscopy. (d) Relative target mRNA levels measured by RT-qPCR and protein levels (e) upon transfection with pEGFP-sp30 for miR-30 family inhibition, or the control vector pEGFP-C1. Data are averages of three biological replicates performed on H9c2 cardiac cells. (f) Target expression levels measured by RT-qPCR following DOX treatment $(1 \mu \mathrm{mol} / \mathrm{l}, 18 \mathrm{~h})$, alone or in combination with miR-30e overexpression (pre-30e transfection). Three independent replicates were performed on H9c2 cells, being presented as averaged ratio to control \pm S.E.M. (g) RT-qPCR data showing expression levels of the predicted miR-30 targets in vivo in DOX-treated hearts ( $15 \mathrm{mg} / \mathrm{kg}$ cumulative dose, $n=5$ rats per group). Student's t-test performed for statistical analysis. Error bars represent \pm S.E.M. 
a direct indicator of $\beta A R$ activation while inversely correlates with $G_{i}$ activity. We observed significantly increased cAMP generation upon acute DOX treatment, as well as following
miR-30 inhibition with our pEGFP-sp30 vector. On the other hand, less cAMP accumulation was detected when overexpressing miR-30e in cardiac cultures (Figure 4a). These
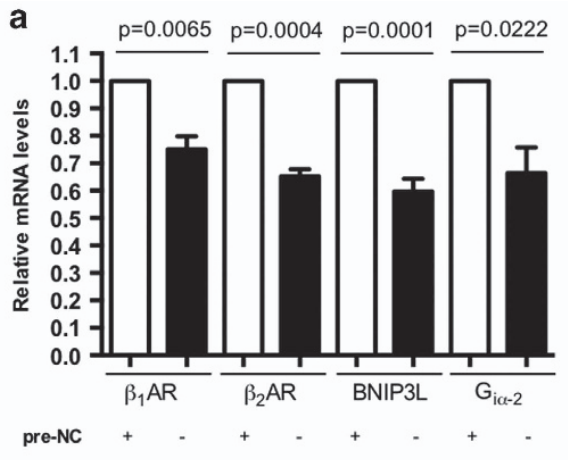

C

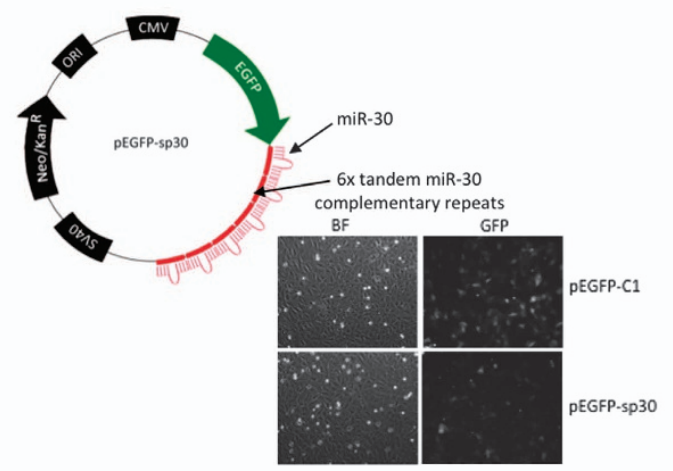

b
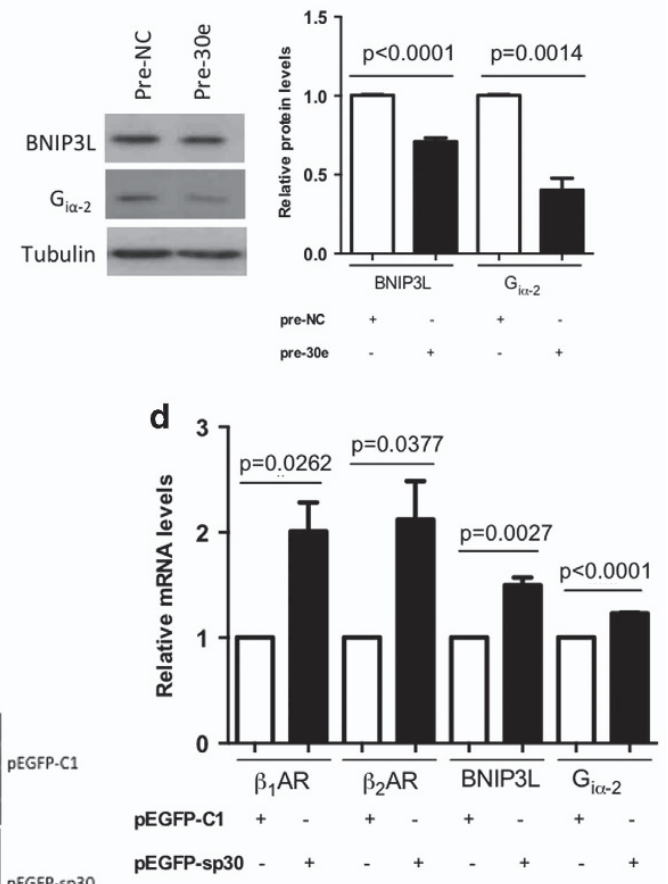

e

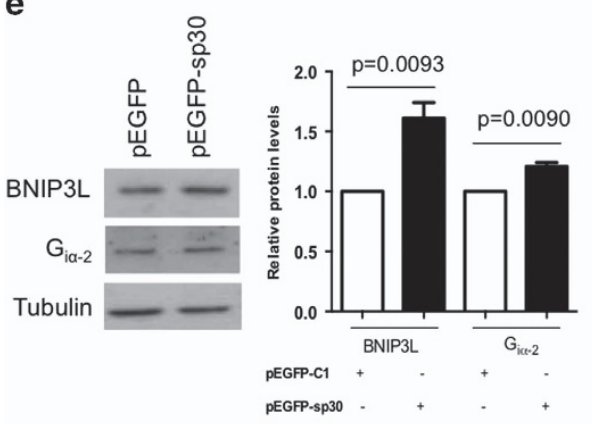

f

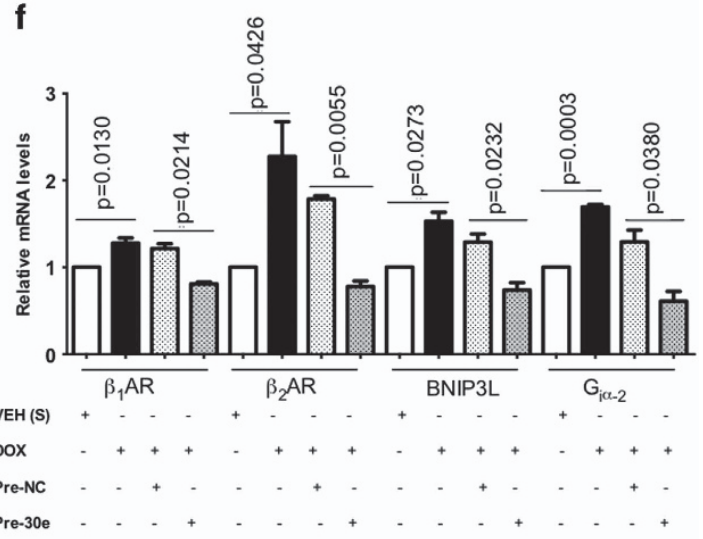

g

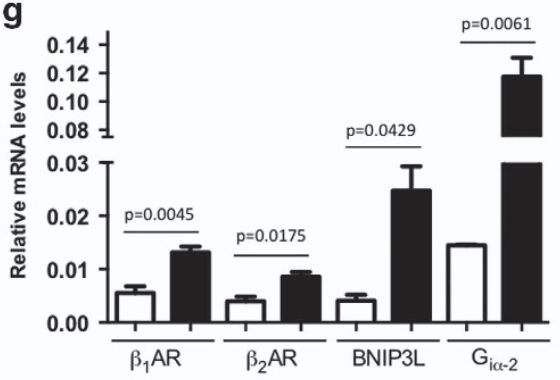

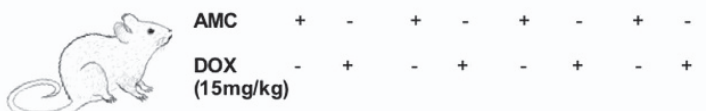


findings are consistent with the regulation of $\beta A R s$ by miR-30 and suggest that miR-30 exerts a greater inhibitory effect on $\beta A R$ s rather than $\mathrm{G}_{\mathrm{ia}-2}$.

Having assessed the alterations that miR-30 expression modulation has on cellular CAMP, we wished to determine the global effects of miR-30 target regulation on cardiomyocyte contractility. We transfected primary isolated ARVCM with miR-30e mimics to achieve overexpression (Supplementary Figure 10) and recorded single-cell contractility using lonOptix. No significant changes were observed in the baseline percentage of contraction amplitude (Figure 4b). We then stimulated transfected ARVCM with increasing concentrations of isoproterenol, a $\beta A R$ agonist, and analyzed paced contractile responses. Cells overexpressing miR-30e showed decreased contractile response to isoproterenol compared with control cells (Figure 4c), a finding supported by the CAMP accumulation data.

miR-30 expression is protective against DOX toxicity. It is known that DOX induces cellular toxicity mediated by caspase-dependent apoptosis. ${ }^{46}$ We investigated whether miR-30 expression modulation is sufficient to affect DOXinduced toxicity in cultured cardiac cells. We measured the activity of caspases 3 and 7 as apoptotic indicator. Ectopically increased miR-30e expression resulted in reduced apoptosis, bringing caspase activity closer to the levels observed upon vehicle treatment (Figure 5a). On the contrary, cultures presented higher caspase activity when inhibiting miR-30 with our pEGFP-sp30 sponge vector (Figure 5a).

DOX has also been described to increase the expression of Bax (pro-apoptotic gene) and to decrease Bcl-2 (antiapoptotic gene) levels. Indeed, elevated Bax/Bcl-2 ratio increases susceptibility to death following apoptotic stimuli ${ }^{47}$ and restored $\mathrm{Bcl}-2$ expression has been shown to protect cardiomyocytes against DOX toxicity. ${ }^{48}$ To further assess the protective effects of miR-30 against DOX insult, we measured the $\mathrm{Bax} / \mathrm{Bcl}-2$ expression ratio in treated cardiac cultures. As expected, DOX treatment progressively increased the Bax/Bcl-2 ratio. In contrast, miR-30e overexpression reduced the Bax/Bcl-2
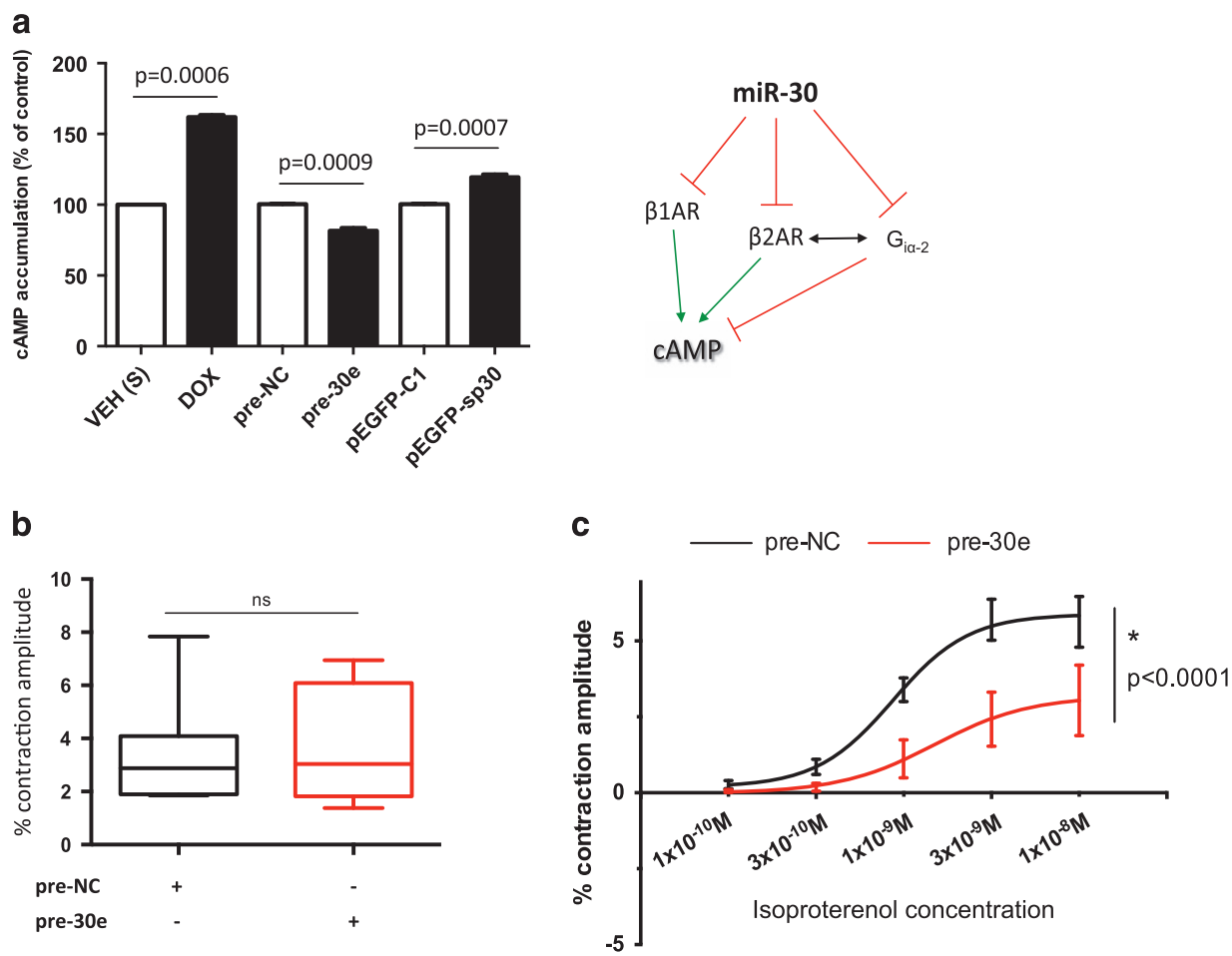

Figure 4 miR-30 effects on cAMP accumulation and contractility. (a) cAMP accumulation data expressed as \% of the respective control samples. Values are the mean \pm S.E.M. of three independent experiments. The diagram on the right shows the contribution to CAMP production of the three miR-30 targets involved in the $\beta$-adrenergic pathway. (b) Baseline $\%$ of contraction amplitude of paced ARVCM transfected with either pre-30e or pre-NC for $48 \mathrm{~h}$ (100 nmol/l), measured using lonOptix ( $n=8-10$ cells from 6 preparations; t-test; ns, not significant). (c) Contractile responses of isoproterenol-stimulated transfected ARVCM, recorded using lonOptix ( $n=6$ cells from 6 preparations, F-test)

Figure 5 High miR-30 levels are protective against DOX insult and reduce ROS generation. (a) Left graph: caspase activity in cardiac cultures treated with DOX alone $(1 \mu \mathrm{mol} / \mathrm{l}, 18 \mathrm{~h})$ and in combination with miR-30e overexpression through pre-30e mimic transfection $(20 \mathrm{nmol} / \mathrm{l})$. Right graph: relative caspase activity upon miR-30 family inhibition using the pEGFP-sp30 sponge vector. (b) Bax and Bcl-2 protein levels measured by western blot analysis. Cells were treated with DOX ( $1 \mu \mathrm{mol} / /, 6 \mathrm{~h}$ and $18 \mathrm{~h})$ and also transfected with pre-30e mimics $(20 \mathrm{nmol} / /)$ in combination with DOX $(1 \mu \mathrm{mo} / / \mathrm{l}, 18 \mathrm{~h})$. Band densitometry was measured using Image J. All values are normalized to Tubulin and expressed as Bax/Bcl-2 ratio. (c) ROS generation of transfected cultures for miR-30 overexpression or inhibition, measured by DCFDA fluorescence intensity. All assays (a, b, c) were performed on $\mathrm{H} 9 \mathrm{c} 2$ cells and results are average of independent triplicates \pm S.E.M. (t-test; ns, not significant) 
ratio upon DOX treatment (Figure 5b). An additional characteristic of DOX-associated cardiomyocyte damage is high ROS levels. ${ }^{49}$ We therefore assessed the impact of miR-30 on
ROS. Notably, cells with high miR-30e expression showed reduced $\mathrm{ROS}$, whereas miR-30 inhibition resulted in higher ROS levels. No further increase in ROS production was a
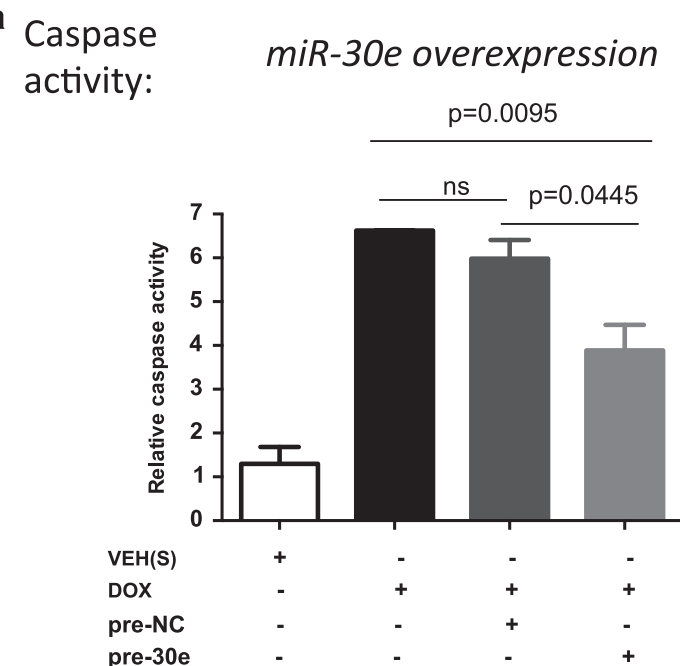

b
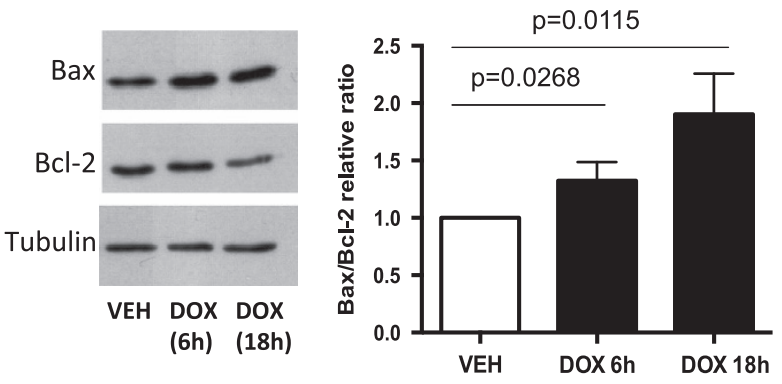

C

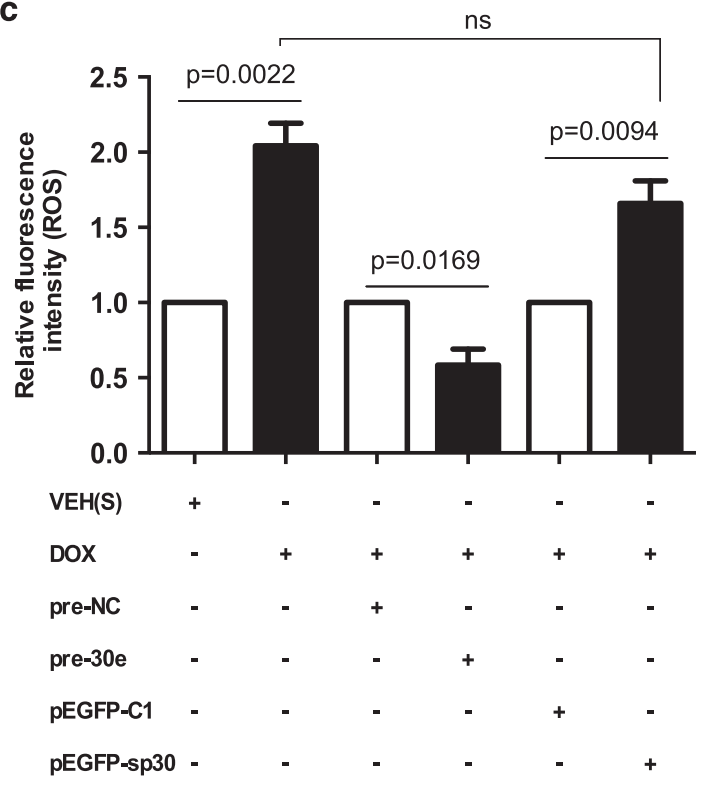

\section{Bax/Bcl-2 ratio}

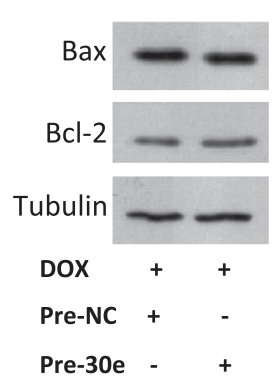

Bax/Bcl-2 ratio

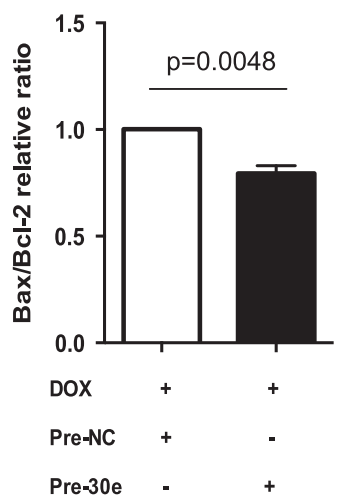


observed when combining DOX treatment and miR-30 inhibition (Figure 5c). This finding further confirmed the benefit of high miR-30 expression in cardiac cells.

GATA-6 mediates DOX-induced miR-30 downregulation. We next wished to establish the mechanism via which DOX downregulates miR-30 expression. GATA-6 is a transcription factor highly expressed in the heart and known to play a key role in cardiac development. ${ }^{50}$ Interestingly, we discovered evidence of GATA- 6 binding to miR-30 cluster promoters by data mining of chromatin immunoprecipitation sequencing experiments, using deepBase (http://deepbase.sysu.edu.cn/). Strikingly, we identified increased GATA-6 levels shortly (30 min-1 h) in primary isolated ARVCM after DOX treatment, accompanied by a sustained reduction in mature miR-30e expression from the $1 \mathrm{~h}$ time point (Figure 6a).

We silenced GATA-6 using RNA interference (Supplementary Figure 12) and, in order to unveil the nature of its regulatory effect, we quantified primary and mature miR-30 levels of two family members coded in different clusters (miR-30d, miR-30e) by RT-qPCR. GATA-6 inhibition resulted in increased levels of miR-30d and miR-30e, at both the primary and mature stages of their bioprocessing (Figure 6b), suggesting an inhibitory role for GATA-6 on the miR-30 promoters. Subsequently, we measured expression levels of the validated miR-30 targets $\left(\beta_{1} A R, \beta_{2} A R, G_{i a-2}\right.$ and BNIP3L) upon GATA-6 silencing, which were accordingly reduced (Figure $6 \mathrm{c}$ ). Decreased target expression levels could be compensated by simultaneous miR-30 family inhibition using LNA oligonucleotides (Figure 6c), suggesting a direct mediation of the GATA- 6 effects on target gene expression by miR-30. Finally, in order to determine the impact of GATA- 6 on cell toxicity, we measured caspase activity in treated $\mathrm{H} 9 \mathrm{c} 2$ cardiac cultures. GATA- 6 inhibition was reduced activation of apoptotic pathways even in the presence of DOX administration (Figure 6d).

\section{Discussion}

We describe here cardiomyocyte-specific alterations in miRNA expression following acute and chronic DOX injury, and post-MI induction. A shared miRNA subset was detected across the assayed models of cardiac toxicity, reflective of a

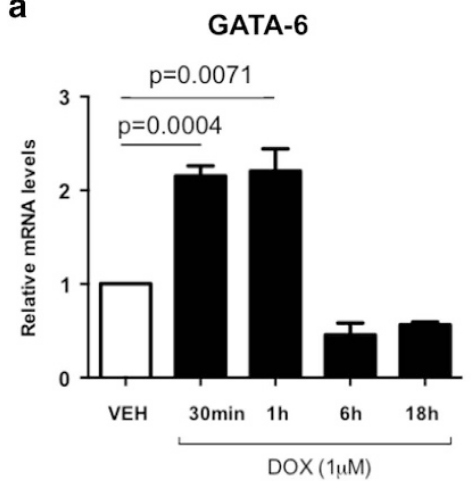

C

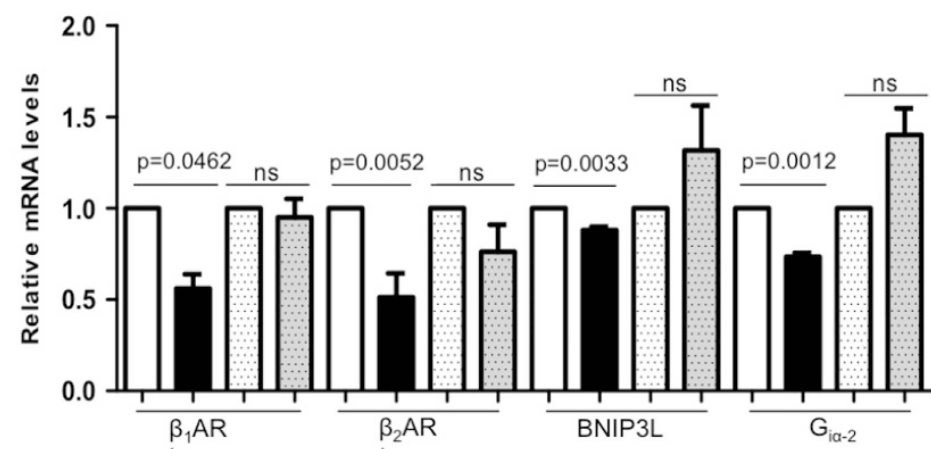

$\operatorname{sinC}$

SiGATA6

LNANC

LNA 30

Figure 6 GATA-6 mediates miR-30 downregulation triggered by DOX. (a) RT-qPCR data showing GATA-6 and miR-30e expression levels in response to DOX time-course treatment $(1 \mu \mathrm{mo} / / /)$. (b) Relative levels of primary and mature miR-30d and miR-30e, upon GATA-6 silencing detected by RT-qPCR. (c) RT-qPCR data for miR-30 target mRNA levels in response to GATA-6 silencing ( $25 \mathrm{nmol} / /, 72 \mathrm{~h}$ ) and after co-transfection with compensatory LNA miR-30 family inhibitors (100 nmol//, $72 \mathrm{~h}$ ). (d) Relative caspase activity in response to GATA-6 silencing, \pm DOX treatment $(1 \mu \mathrm{mol} / \mathrm{l}, 18 \mathrm{~h})$. All values $(\mathbf{a}, \mathbf{b}, \mathbf{c}, \mathbf{d})$ were obtained performing biological triplicates on H9c2 cells and are presented as averaged ratio to the respective control \pm S.E.M. All RT-qPCR results were normalized to the correspondent U6 Ct value (ns, not significant; $t$-test)

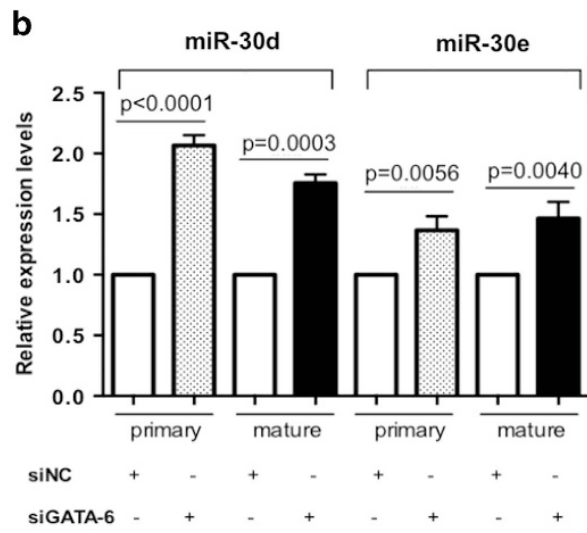

d

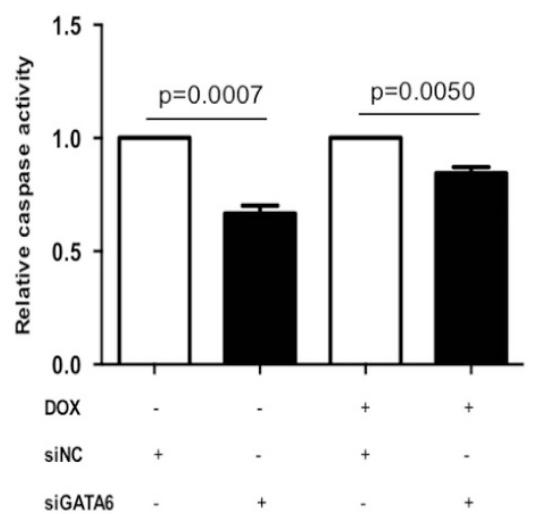


consistencies in the cardiomyocyte response to injury. However, those miRNAs only differentially expressed in response to acute and/or sustained DOX treatments also suggest the existence of unique molecular signatures in anthracycline-related myocardial damage (Figure 1b). We believe that the rapid alteration of these miRNAs within hours of exposure to DOX and maintained over time could be of translational relevance, both as diagnostic biomarker and as therapeutic strategy.

In particular, we describe here the myocardial downregulation of several miR-30 family members by acute and sustained DOX treatment and validate four essential genes for cardiomyocyte biology as novel miR-30 targets $\left(\beta_{1} A R, \beta_{2} A R, G_{i a-2}\right.$ and BNIP3L/NIX; Figures $2 c$ and 3 ). These data are consistent with our previous findings in which miRNAs often target multiple members of a shared signaling pathway, as individual target inhibition in vivo is unlikely to be complete. ${ }^{51}$ Alterations in several of these genes have previously been linked to HF (i.e., desensitized $\beta A R s$, chronic $\beta_{1} A R$ activation and increased $\mathrm{G}_{\mathrm{ia}-2}$ expression). ${ }^{9,10}$ Moreover, the pro-apoptotic target gene BNIP3L/NIX has been linked to cardiomyopathy through mitochondrial cell death. ${ }^{39,52}$ In concordance with the present data (Figure $3 \mathrm{~g}$ ), enhanced $\beta_{2} \mathrm{AR}$ cardiac expression upon DOX treatment in vivo has recently been reported, although the mechanisms involved were not investigated. ${ }^{53}$ Moreover, $\beta_{2} A R$ deletion has been shown to prevent cardiomyopathy. ${ }^{54}$

Reduced miR-30 levels have been associated with myocardial matrix remodeling and with angiotensin II-induced hypertrophy. ${ }^{31,55}$ Low miR-30e expression was also detected in myocardial tissue from patients with dilated cardiomyopathy and aortic stenosis. ${ }^{56}$ However, the specific myocardial expression of miR-30 in the context of exposure to DOX and its downstream mechanisms had not been previously demonstrated. We show that high miR-30 levels are connected to a global decrease in cAMP (Figure 4a), likely to be advantageous given the described link between high cAMP/PKA and cardiomyocyte death. ${ }^{57}$ The greater accumulation of cAMP triggered by acute DOX treatment than by specific miR-30 inhibition alone raises the hypothesis that DOX may be acting through alternate pathways toward the same detrimental effect. Upon miR-30e overexpression, ARVCM presented decreased contractile responses to isoproterenol stimulation. As $\beta A R$ and $G_{i a-2}$ have opposing effects on the same pathway, such attenuated contractile response reveals a phenotype suggesting preferential $\beta A R$ repression alongside a compensatory inhibition of $\mathrm{G}_{\mathrm{ia}-2}$ (Figures $4 \mathrm{a}$ and $\mathrm{c}$, Supplementary Figure 11). This negative feedback loop is likely to contribute to the fine-tuning of the system, which is characteristic of miRNAs and allows intensive regulation of complex biological processes. ${ }^{58}$ The preferential $\beta A R$ inhibition observed results in an overall effect simulating $\beta$-blocker agents, which are commonly used to treat $\mathrm{HF}$. Furthermore, milder $\mathrm{G}_{\mathrm{i \alpha -2}}$ targeting by miR-30 could also tentatively be beneficial, as retention of $\mathrm{G}_{\mathrm{i}}$ activity serves to reduce myocyte death. ${ }^{59}$

For the first time, we show that DOX treatment triggers an early induction of GATA-6 expression. In addition, our data suggest that DOX-induced GATA-6 upregulation represses miR-30 transcription, a model supported by (primary and mature) miR-30 upregulation in response to GATA-6 silencing.
In keeping with this, miR-30-dependent target downregulation was observed upon GATA-6 inhibition (Figure 6).

Cardiomyocyte apoptosis, involving cytochrome $\mathrm{c}$ and caspase activation, is a crucial event in HF development in general and after DOX injury. ${ }^{46,60,61}$ Importantly, high miR-30 levels are protective against DOX insult while inhibition of miR-30 is sufficient to increase caspase-mediated apoptosis (Figure 5a). Consistent with this, the $\mathrm{Bax} / \mathrm{Bcl}-2$ ratio was lower in cardiac cultures overexpressing miR-30e upon DOX administration (Figure $5 \mathrm{~b}$ ) and ROS generation inversely correlated with miR-30 levels, further indicating the beneficial effects of miR-30 expression for cardiac cell viability (Figure 5c).

Interestingly, $\beta$-blockers have been shown to prevent cancer progression both in patient cohorts ${ }^{62,63}$ and animal models. ${ }^{64-66}$ Moreover, increasing evidence indicates a tumor-suppressor role for miR-30, having been shown to minimize resistance to chemotherapy and to correlate with less invasive tumors. ${ }^{67-70}$ We confirmed this finding in our system by Transwell migration assays performed on the highly metastatic breast cancer cell line MDA-MB-231, which had impaired migration upon miR-30 overexpression (Supplementary Figure 13). In addition, analyses of breast cancer patients' data freely available at the Gene Expression Omnibus (http://www.ncbi.nlm.nih.gov/geo/) consistently showed a reduction in miR-30 levels compared with control individuals (Supplementary Figure 14). Similarly, both SurvMicro (http://bioinformatica.mty.itesm.mx/SurvMicro) and PROGmiR (http://www.compbio.iupui.edu/progmir) prognostic databases indicated high miR-30 expression to be a biomarker of good prognosis in breast cancer (Supplementary Figure 15). These data are encouraging and suggest that controlled administration of miR-30 mimics could deliver a pleiotropic therapeutic effect. Unfortunately, we could not carry out the required experiments to investigate this further due to the restrictions applied on animal experimentation by the UK Home Office.

In aggregate, our findings should contribute to unraveling the complex web of mechanisms via which DOX is able to cause cardiac toxicity and dysfunction, as we reveal the targeting of pathways involved in cardiomyocyte function and viability by a miRNA family that is altered by DOX (Figure 7).

\section{Materials and Methods}

Animal models. All animal surgical procedures and perioperative management were carried out in accordance with the United Kingdom Home Office Guide on the Operation of Animals (Scientific Procedures) Act 1986 by licensed personnel. See Supplementary Information for details of the experimental procedures.

MI induction: Adult male Sprague-Dawley rats (250-300 g) underwent proximal LAD coronary artery ligation to induce chronic $\mathrm{Ml}$ as previously described by our group. ${ }^{28}$ Animals were anesthetized with $5 \%$ isofluorane in $\mathrm{O}_{2}$ and maintained on $1.5 \%$ isofluorane during surgery. $0.05 \%$ buprenorphine and $5 \mathrm{mg} / \mathrm{kg}$ enrofloxacil were administered intraperitoneally to prevent pain and infection. Two infarcted cohorts were generated. To model early post MI-hypertrophy, animals were killed 4 weeks after Ml induction $(n=5)$. Late-stage $\mathrm{HF}$ model hearts were explanted and prepared for cell isolation 16-20 weeks post Ml surgery $(n=5)$, when an advanced HF phenotype was established. ${ }^{28}$ Age-matched controls (AMCs, $n=5$ for each) were used in both cases to preserve animal welfare. 


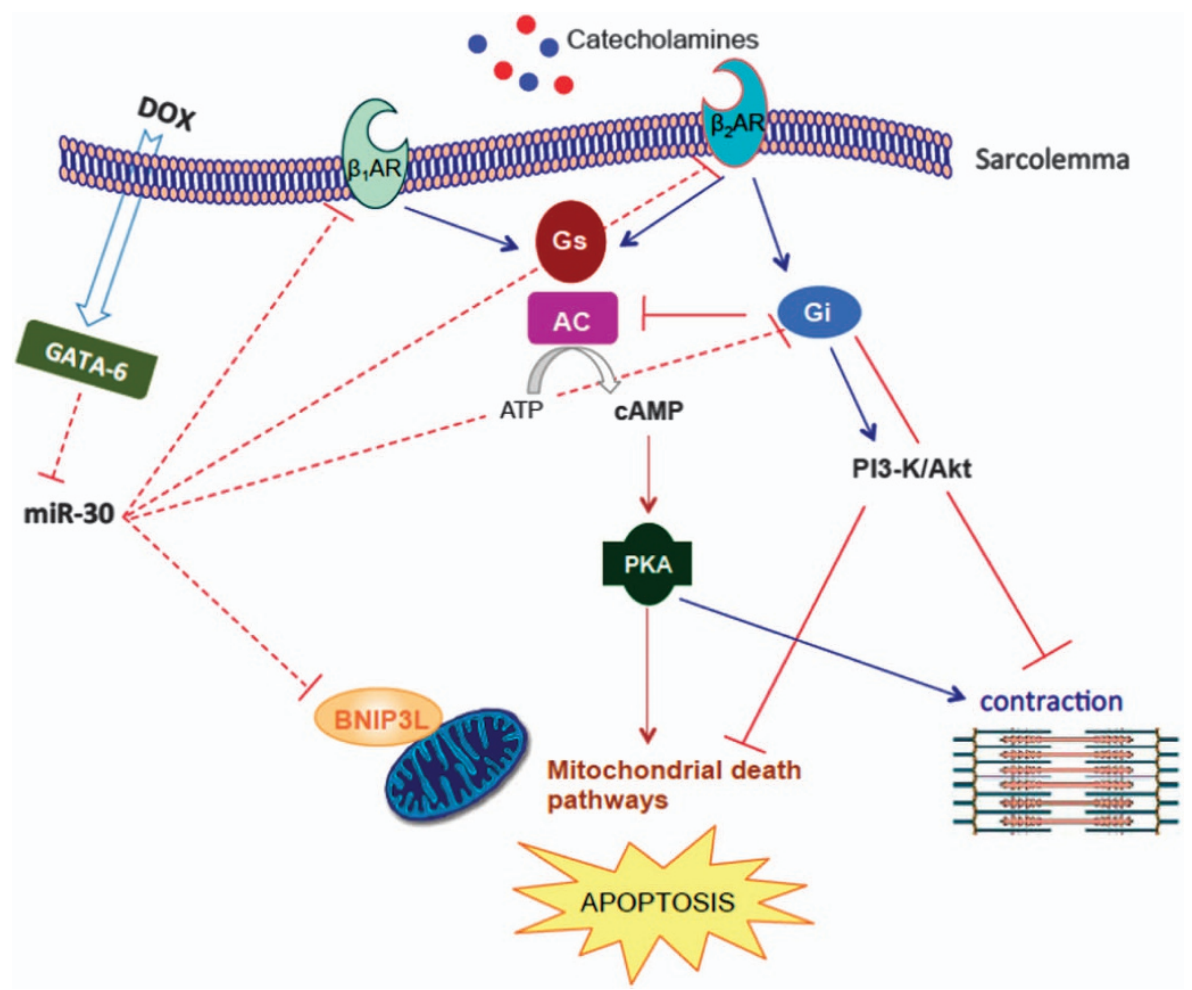

Figure 7 Proposed model: importance of the miR-30 regulatory effects in cardiomyocytes following DOX exposure. Upon DOX treatment, there seems to be an acute increase of GATA-6 expression in cardiomyocytes. GATA-6 is able to repress miR-30 family expression, resulting in DOX-induced miR-30 downregulation. In turn, the expression levels of miR-30 targets are upregulated. Three of the miR-30 target genes are involved in the complex catecholamine/adrenergic pathway. A correct equilibrium of members is crucial in this signaling cascade, as AC-cAMP-PKA activation promotes cardiomyocyte contraction, whereas Gi impairs contraction but protects against apoptosis via Phosphoinositide 3-kinase/Protein kinase B. In addition, miR-30 represses BNIP3L, which is involved in mitochondrial death. miR-30 expression seems to be essential in regulating mitochondrial apoptotic pathways and it is able to partially counteract DOX-induced toxicity. Solid lines: previously described mechanisms, dashed lines: novel mechanisms

DOX-induced HF model: Adult male Sprague-Dawley rats (250-300 g) were administered a total cumulative dose of $15 \mathrm{mg} / \mathrm{kg}$ of DOX delivered via six intraperitoneal injections over 2 weeks $(n=5)$. Each injection contained $2.5 \mathrm{mg} / \mathrm{kg}$ of DOX (Teva, Castleford, UK) diluted in saline up to a volume of $2 \mathrm{ml}$. AMC rats were used as controls $(n=5)$. Animals were kept under constant monitoring for signs of HF and any other possible adverse effects. Three weeks after the last injection, animals were killed and hearts explanted, weighed and prepared for cell isolation.

Ventricular cardiomyocyte isolation. Ventricular cardiomyocyte isolation from adult Sprague-Dawley rat hearts was carried out via serial enzymatic collagenase-hyaluronidase digestion as described previously ${ }^{28}$ (see Supplementary Information).

Cell culture and treatments. Adult rat ventricular cardiomyocytes (ARVCM) were maintained in supplemented M199 (Invitrogen, Paisley, UK). ARVCM from AMC animals or DOX/4week post-MI models were plated in laminin (Invitrogen)coated six-well plates and viable rod-shaped cells were allowed to attach for an hour. Apoptotic floating ARVCM were then discarded. For in vitro studies, medium was renewed and DOX (Teva) was then administered to cultured viable ARVCM. A clinically relevant concentration of $1 \mu \mathrm{mol} / / \mathrm{l}$ was used throughout. ${ }^{6}$ This dose was allowed to act in the cultures for $6 \mathrm{~h}$ for miRNA expression level check. For evaluation of gene expression changes (mRNA and protein), DOX treatments were conducted over $18 \mathrm{~h}$. Control wells were treated with the same volume of vehicle (saline). H9c2 cardiac muscle cells were used as model to overcome the limitations of ARVCM, ${ }^{41}$ and were maintained in Dulbecco's modified Eagle's medium supplemented with $10 \%$ fetal calf serum, $1 \%$ penicillin/streptomycin and $2 \%$ L-glutamine.
Transfections. H9c2 cells were plated at $40 \%$ confluence in six-well plates and transfected on the following day and for $72 \mathrm{~h}$, with $20 \mathrm{nmol} / \mathrm{l}$ of precursor miRNAs for mimicry overexpression (pre-30e or pre-NC; Applied Biosystems, Paisley, UK) and $100 \mathrm{nmol} / /$ of miRCURY LNA for family inhibition (Exiqon, Vedbaek, Denmark), using HiPerFect transfection reagent (Qiagen, West Sussex, UK). For sponge vector transfections (pEGFP-sp30 or pEGFP-C1 control), we used Lipofectamine 2000 (Life Technologies Ltd, Paisley, UK) on $90 \%$ confluent cultures and allowed them to get transfected for $48 \mathrm{~h}$ in antibiotic-free medium. For luciferase assays, H9c2 cells were plated at a density of 50000 cells/well in 24-well plates and co-transfected the following day with $100 \mathrm{nmol} / \mathrm{I}$ of pre-30e/pre-NC and $100 \mathrm{ng}$ of the respective pLightSwitch_3'UTR GoClone for each target 3'UTR, also using Lipofectamine 2000. In each independent replicate, three wells were transfected for each condition. For contractility studies, primary isolated ARVCM were transfected in six-well plates and kept in solution for $48 \mathrm{~h}$. We used Lipofectamine 2000 (Life Technologies Ltd) and a molarity of $100 \mathrm{nmol} / \mathrm{l}$ of pre-30e or pre-NC.

Sponge plasmid construction. The pEGFP-sp30 sponge vector was manufactured by annealing, purifying and cloning primers coding for six bulged tandem repeats of mismatched miR-30-binding sites. The insert was cloned into the pEGFP-C1 plasmid (Clontech, Mountain View, CA, USA) using the Hindlll and BamHI restriction sites, as 3'UTR of the EGFP-coding sequence. The generated sponge vector was then verified by sequencing. Primers used for sponge vector construction are described in Supplementary Table 1.

miRNA profiling. miRNA profiling was performed using nCounter Rat miRNA Expression Assay (NanoString Technology, Seattle, WA, USA), using $150 \mathrm{ng}$ of total RNA. Biological triplicates were assayed for all conditions. Background removal was performed following the producer's instructions and quantile normalization of raw data was carried out using Partek Genomic suite (St. Louis, MO, USA). A list of the 20 most significantly dysregulated hits for each model including $P$ values and fold 
changes can be observed in Supplementary Table 3. The full list of assayed miRNA sequences and complete raw data has been deposited at Gene Expression Omnibus (GSE36239).

3'UTR Luciferase assays. After $24 \mathrm{~h}$ of co-transfection, cells were lysed using passive lysis buffer (Promega, Madison, WI, USA) and luciferase activity was detected using LightSwitch Assay System reagent (Switchgear Genomics, Menlo Park, CA, USA), in a GLOMAX 96 Microplate luminometer (Promega). The 3'UTRLuc vectors used were $\beta_{1}$ AR (S812620), $\beta_{2}$ AR (S804637), BNIP3L (S812956) and $G_{\mathrm{i}_{\alpha-2}}$ (S807754), all from Switchgear Genomics. For 3'UTR site-directed mutagenesis of these vectors, we used the QuickChange II or II XL Site-Directed Mutagenesis Kit (Agilent Technology, Edinburgh, UK) according to the manufacturer's instructions. The sequences of the plasmid used for this protocol are reported in Supplementary Table 2. All resulting plasmids were verified by sequencing.

cAMP accumulation. Treated $\mathrm{H} 9 \mathrm{c} 2$ cells were lysed using $0.1 \mathrm{~mol} / \mathrm{HCl}$. The lysates were then assayed using the 96-well strip cAMP EIA kit (Cayman Chemical, Ann Arbor, MI, USA) and analyzed according to the manufacturer's instructions. At least duplicate wells were included for each sample to account for technical variability.

Caspase assays. Apoptosis in treated $\mathrm{H} 9 \mathrm{c} 2$ cultures was measured by quantifying caspase 3 and caspase 7 activities, according to the manufacturers' instructions (Caspase-Glo 3/7 Assay, Promega). Briefly, both medium and cells were collected from each well and $50 \mu \mathrm{l}$ of cell suspension were transferred to a 96-well plate in duplicate. $100 \mu$ of Caspase-Glo reagent were then mixed into each well an incubated for $1 \mathrm{~h}$ at room temperature, before reading luminescence intensity using a PHERAstar ${ }^{\text {Plus }}$ machine (BMG Labtech, Aylesbury, UK). Luminescence value for caspase activity was normalized to protein content.

ROS detection. Levels of ROS generation in the cultures were measured by using the MitoScience DCFDA Cellular ROS Detection Assay Kit (Abcam Plc., Cambridge, UK), according to the manufacturer's instructions. Fluorescence readings were performed in 96-well microplates, using a PHERAstar ${ }^{\text {Plus }}$ machine.

Contractility of transfected ARVCM. Following $48 \mathrm{~h}$ of pre-miR-30e/NC transfection, ARVCM in suspension were transferred into a Perspex bath using a glass coverslip as base. Cells were superfused with $\mathrm{KH}$ solution $\left(1 \mathrm{mmo} / / \mathrm{Ca}^{2+}\right)$ at $37{ }^{\circ} \mathrm{C}$, equilibrated with $95 \% \mathrm{O}_{2} / 5 \% \mathrm{CO}_{2}$. An inverted microscope was used for imaging and myocytes were electrically stimulated at $0.5 \mathrm{~Hz}$, measuring cell shortening with the lonOptix system. Values were presented as \% contraction amplitude. Cardiomyocytes from six different preparations were used for each treatment.

Statistical analysis. Unless differently stated, data are presented as mean \pm S.E.M. calculated using Prism 5 (GraphPad Software Inc.) and Student's $t$-test was used for value comparison, being $P<0.05$ considered as significance threshold. To analyze the contractile responses of ARVCM to increasing concentrations of isoproterenol, $\mathrm{F}$ tests were performed.

\section{Conflict of Interest}

The authors declare no conflict of interest.

Acknowledgements. We thank Mr. Peter O'Gara for cardiomyocyte cell isolation. This work was supported by the British Heart Foundation (BHF) [WSCC G00000 164104 (LRA) and Intermediate Clinical Research Fellowship (ARL)], the Wellcome Trust (MBS), the Imperial College Trust and Healthcare Charity, Action Against Cancer, the NIHR and the AICR along with the Imperial ECMC and BRC. The funders had no role in the study design, data collection and analysis, decision to publish or preparation of the manuscript.

1. Weiss RB. The anthracyclines: will we ever find a better doxorubicin? Semin Oncol 1992; 19 670-686.

2. Gewirtz D. A critical evaluation of the mechanisms of action proposed for the antitumor effects of the anthracycline antibiotics adriamycin and daunorubicin. Biochem Pharmacol 1999; 57 : 727-741.
3. Hjalmarson A, Waagstein $F$. The role of [beta]-blockers in the treatment of cardiomyopathy and ischaemic heart failure. Drugs 1994; 47: 31-40.

4. Davies KJ, Doroshow JH. Redox cycling of anthracyclines by cardiac mitochondria. I. Anthracycline radical formation by NADH dehydrogenase. J Biol Chem 1986; 261: 3060-3067.

5. Velez JM, Miriyala S, Nithipongvanitch R, Noel T, Plabplueng CD, Oberley $T$ et al. p53 Regulates oxidative stress-mediated retrograde signaling: a novel mechanism for chemotherapy-induced cardiac injury. PLoS ONE 2011; 6: e18005.

6. Minotti G, Menna P, Salvatorelli E, Cairo G, Gianni L. Anthracyclines: molecular advances and pharmacologic developments in antitumor activity and cardiotoxicity. Pharmacol Rev 2004; 56: 185-229.

7. Zhang S, Liu X, Bawa-Khalfe T, Lu L-S, Lyu YL, Liu LF et al. Identification of the molecular basis of doxorubicin-induced cardiotoxicity. Nat Med 2012; 18: 1639-1642.

8. Engelhardt S, Hein L, Wiesmann F, Lohse MJ. Progressive hypertrophy and heart failure in B1-adrenergic receptor transgenic mice. Proc Natl Acad Sci USA 1999; 96: 7059-7064.

9. Lohse MJ, Engelhardt $S$, Eschenhagen T. What is the role of $\beta$-adrenergic signaling in heart failure? Circ Res 93: 896-906.

10. Eschenhagen T, Mende U, Nose M, Schmitz W, Scholz H, Haverich A et al. Increased messenger RNA level of the inhibitory $G$ protein alpha subunit $G i$ alpha-2 in human end-stage heart failure. Circ Res 1992; 70: 688-696.

11. Feldman AM, Cates AE, Veazey WB, Hershberger RE, Bristow MR, Baughman KL et al. Increase of the $40,000-\mathrm{mol}$ wt pertussis toxin substrate (G protein) in the failing human heart. J Clin Invest 1988; 82: 189-197.

12. Eichhorn EJ. The paradox of $\beta$-adrenergic blockade for the management of congestive heart failure. Am J Med 1992; 92: 527-538.

13. Waagstein $F$, Hjalmarson $A$, Varnauskas $E$, Wallentin I. Effect of chronic beta-adrenergic receptor blockade in congestive cardiomyopathy. Br Heart J 1975; 37: 1022-1036.

14. Bartel D. MicroRNAs: target recognition and regulatory functions. Cell 2009; 136: 215-233.

15. Marco A, Ninova M, Ronshaugen M, Griffiths-Jones S. Clusters of microRNAs emerge by new hairpins in existing transcripts. Nucleic Acids Res 2013; 41: 7745-7752.

16. Bartel D. MicroRNAs: genomics, biogenesis, mechanism, and function. Cell 2004; 116 : 281-297.

17. Lewis BP, Burge CB, Bartel DP. Conserved seed pairing, often flanked by adenosines, indicates that thousands of human genes are microRNA targets. Cell 2005; 120: $15-20$.

18. Chen J-F, Murchison EP, Tang R, Callis TE, Tatsuguchi M, Deng Z et al. Targeted deletion of Dicer in the heart leads to dilated cardiomyopathy and heart failure. Proc Natl Acad Sci USA 2008; 105: 2111-2116.

19. da Costa Martins PA, Bourajjaj M, Gladka M, Kortland M, van Oort RJ, Pinto YM et al. Conditional dicer gene deletion in the postnatal myocardium provokes spontaneous cardiac remodeling. Circulation 2008; 118: 1567-1576.

20. Zhao Y, Ransom JF, Li A, Vedantham V, von Drehle M, Muth AN et al. Dysregulation of cardiogenesis, cardiac conduction, and cell cycle in mice lacking miRNA-1-2. Cell 2007; 129: 303-317.

21. van Rooij E. A signature pattern of stress-responsive microRNAs that can evoke cardiac hypertrophy and heart failure. Proc Natl Acad Sci USA 2006; 103: 18255-18260.

22. Thum T, Galuppo P, Wolf C, Fiedler J, Kneitz S, van Laake LW et al. MicroRNAs in the Human Heart. Circulation 2007; 116: 258-267.

23. van Rooij E, Sutherland L, Qi X, Richardson J, Hill J, Olson E. Control of stress-dependent cardiac growth and gene expression by a microRNA. Science 2007; 316: 575-579.

24. Dong D-L, Chen C, Huo R, Wang N, Li Z, Tu Y-J et al. Reciprocal repression between microRNA-133 and calcineurin regulates cardiac hypertrophy. Hypertension 2010; 55 : 946-952.

25. Horie T, Ono K, Nishi H, Nagao K, Kinoshita M, Watanabe S et al. Acute doxorubicin cardiotoxicity is associated with miR-146a-induced inhibition of the neuregulin-ErbB pathway. Cardiovasc Res 2010; 87: 656-664.

26. Vacchi-Suzzi C, Bauer Y, Berridge BR, Bongiovanni S, Gerrish K, Hamadeh HK et al. Perturbation of microRNAs in Rat Heart during Chronic Doxorubicin Treatment. PLoS One 2012; 7: e40395

27. Siveski-lliskovic N, Kaul N, Singal P. Probucol promotes endogenous antioxidants and provides protection against adriamycin-induced cardiomyopathy in rats. Circulation 1994; 89: 2829-2835.

28. Lyon AR, MacLeod KT, Zhang Y, Garcia E, Kanda GK, Lab MJ et al. Loss of T-tubules and other changes to surface topography in ventricular myocytes from failing human and rat heart. Proc Natl Acad Sci 2009; 106: 6854-6859.

29. van Rooij E, Sutherland LB, Thatcher JE, DiMaio JM, Naseem RH, Marshall WS et al. Dysregulation of microRNAs after myocardial infarction reveals a role of miR-29 in cardiac fibrosis. Proc Natl Acad Sci USA 2008; 105: 13027-13032.

30. Thum T, Gross C, Fiedler J, Fischer T, Kissler S, Bussen M et al. MicroRNA-21 contributes to myocardial disease by stimulating MAP kinase signalling in fibroblasts. Nature 2008; 456 : 980-984.

31. Duisters RF, Tijsen AJ, Schroen B, Leenders JJ, Lentink V, van der Made I et al. miR-133 and miR-30 regulate connective tissue growth factor. Circ Res 104: 170-178.

32. Care A, Catalucci D, Felicetti F, Bonci D, Addario A, Gallo P et al. MicroRNA-133 controls cardiac hypertrophy. Nat Med 2007; 13: 613-618.

33. Hu S, Huang M, Li Z, Jia F, Ghosh Z, Lijkwan MA et al. MicroRNA-210 as a novel therapy for treatment of ischemic heart disease. Circulation 2010; 122: S124-S131. 
34. Shieh JTC, Huang Y, Gilmore J, Srivastava D. Elevated miR-499 levels blunt the cardiac stress response. PLOS One 2011; 6: e19481.

35. Wang G-K, Zhu J-Q, Zhang J-T, Li Q, Li Y, He J et al. Circulating microRNA: a novel potential biomarker for early diagnosis of acute myocardial infarction in humans. Eur Heart $J$ 31: 659-666.

36. Lewis B, Burge C, Bartel D. Conserved seed pairing, often flanked by adenosines, indicates that thousands of human genes are microRNA targets. Cell 2005; 120: 15-20.

37. Chen Y, Lewis W, Diwan A, EH-Y Cheng, Matkovich SJ, Dorn GW. Dual autonomous mitochondrial cell death pathways are activated by Nix/BNip3L and induce cardiomyopathy. Proc Natl Acad Sci USA 2010; 107: 9035-9042.

38. Ding W-X, Ni H-M, Li M, Liao Y, Chen X, Stolz DB et al. Nix is critical to two distinct phases of mitophagy, reactive oxygen species-mediated autophagy induction and parkin-ubiquitinp62-mediated mitochondrial priming. J Biol Chem 2010; 285: 27879-27890.

39. Diwan A, Wansapura J, Syed FM, Matkovich SJ, Lorenz JN, Dorn GW. Nix-Mediated apoptosis links myocardial fibrosis, cardiac remodeling, and hypertrophy decompensation. Circulation 117: 396-404.

40. Yussman MG, Toyokawa T, Odley A, Lynch RA, Wu G, Colbert MC et al. Mitochondrial death protein Nix is induced in cardiac hypertrophy and triggers apoptotic cardiomyopathy. Nat Med 2002; 8: 725-730.

41. L'Ecuyer T, Horenstein MS, Thomas R, Vander Heide R. Anthracycline-induced cardiac injury using a cardiac cell line: potential for gene therapy studies. Mol Genetics Metab 2001; 74: 370-379.

42. Hescheler J, Meyer R, Plant S, Krautwurst D, Rosenthal W, Schultz G. Morphological, biochemical, and electrophysiological characterization of a clonal cell (H9c2) line from rat heart. Circ Res 1991; 69: 1476-1486.

43. Hamdani N, Velden J. Lack of specificity of antibodies directed against human betaadrenergic receptors. Naunyn Schmiedeberg Arch Pharmacol 2009; 379: 403-407.

44. Pradidarcheep W, Stallen J, Labruyère W, Dabhoiwala N, Michel M, Lamers W. Lack of specificity of commercially available antisera against muscarinergic and adrenergic receptors. Naunyn Schmiedeberg Arch Pharmacol 2009; 379: 397-402.

45. Ebert MS, Neilson JR, Sharp PA. MicroRNA sponges: competitive inhibitors of small RNAs in mammalian cells. Nat Meth 2007; 4: 721-726.

46. Childs AC, Phaneuf SL, Dirks AJ, Phillips T, Leeuwenburgh C. Doxorubicin treatment in vivo causes cytochrome $\mathrm{C}$ release and cardiomyocyte apoptosis, as well as increased mitochondrial efficiency, superoxide dismutase activity, and Bcl-2:Bax Ratio. Cancer Res 2002; 62: 4592-4598.

47. Oltval $\mathrm{ZnN}$, Milliman $\mathrm{CL}$, Korsmeyer SJ. Bcl-2 heterodimerizes in vivo with a conserved homolog, Bax, that accelerates programed cell death. Cell 1993; 74: 609-619.

48. Lv X, Yu X, Wang Y, Wang F, Li H, Wang Y et al. Berberine inhibits doxorubicin-triggered cardiomyocyte apoptosis via attenuating mitochondrial dysfunction and increasing $\mathrm{Bcl}-2$ expression. PLoS One 2012; 7: e47351.

49. Mukhopadhyay P, Rajesh M, Bátkai S, Kashiwaya $Y$, Haskó G, Liaudet L et al. Role of superoxide, nitric oxide, and peroxynitrite in doxorubicin-induced cell death in vivo and in vitro. Am J Physiol Heart Circ Physiol 2009; 296: H1466-H1483.

50. Molkentin JD. The zinc finger-containing transcription factors GATA-4, -5 , and -6 : ubiquitously expressed regulators of tissue-specific gene expression. J Biol Chem 2000; 275: 38949-38952.

51. Frampton AE, Castellano L, Colombo T, Giovannetti E, Krell J, Jacob J et al. MicroRNAs cooperatively inhibit a network of tumor suppressor genes to promote pancreatic tumor growth and progression. Gastroenterology 2014; 146: 268-277 e18.

52. Chen Y, Lewis W, Diwan A, Cheng EHY, Matkovich SJ, Dorn GW. Dual autonomous mitochondrial cell death pathways are activated by Nix/BNip3L and induce cardiomyopathy. Proc Natl Acad Sci 2010; 107: 9035-9042.

53. Merlet N, Piriou N, Rozec B, Grabherr A, Lauzier B, Trochu J-N et al. Increased beta2adrenoceptors in doxorubicin-induced cardiomyopathy in rat. PLoS One 2013; 8: e64711.
54. Fajardo G, Zhao M, Urashima T, Farahani S, Hu D-Q, Reddy S et al. Deletion of the $\beta 2$ adrenergic receptor prevents the development of cardiomyopathy in mice. J Mol Cell Cardiol 2013; 63: 155-164.

55. Pan W, Zhong Y, Cheng C, Liu B, Wang L, Li A et al. MiR-30-regulated autophagy mediates angiotensin II-induced myocardial hypertrophy. PLoS One 2013; 8: e53950.

56. Ikeda S, Kong SW, Lu J, Bisping E, Zhang H, Allen PD et al. Altered microRNA expression in human heart disease. Physiol Genomics 2007; 31: 367-373.

57. Zhu W-Z, Wang S-Q, Chakir K, Yang D, Zhang T, Brown JH et al. Linkage of $\beta 1$-adrenergic stimulation to apoptotic heart cell death through protein kinase A,Äindependent activation of Ca2+/calmodulin kinase II. J Clin Invest 2003; 111: 617-625.

58. van Rooij E, Marshall WS, Olson EN. Toward MicroRNA-Based Therapeutics for Heart Disease: The Sense in Antisense. Circ Res 2008; 103: 919-928.

59. Zhu W-Z, Zheng M, Koch WJ, Lefkowitz RJ, Kobilka BK, Xiao R-P. Dual modulation of cell survival and cell death by $\beta 2$-adrenergic signaling in adult mouse cardiac myocytes. Proc Natl Acad Sci USA 2001; 98: 1607-1612.

60. Green PS, Leeuwenburgh C. Mitochondrial dysfunction is an early indicator of doxorubicininduced apoptosis. Biochim Biophys Acta 2002; 1588: 94-101.

61. Narula J, Pandey P, Arbustini E, Haider N, Narula N, Kolodgie FD et al. Apoptosis in heart failure: Release of cytochrome $\mathrm{c}$ from mitochondria and activation of caspase- 3 in human cardiomyopathy. Proc Natl Acad Sci USA 1999; 96: 8144-8149.

62. Barron TI, Connolly RM, Sharp L, Bennett K, Visvanathan K. Beta blockers and breast cancer mortality: a population- based study. J Clin Oncol 2011; 29: 2635-2644.

63. Melhem-Bertrandt A, Chavez-MacGregor M, Lei X, Brown EN, Lee RT, Meric-Bernstam F et al. Beta-Blocker use is associated with improved relapse-free survival in patients with triplenegative breast cancer. J Clin Oncol 2011; 29: 2645-2652.

64. Hassan S, Karpova Y, Baiz D, Yancey D, Pullikuth A, Flores A et al. Behavioral stress accelerates prostate cancer development in mice. J Clin Invest 2013; 123: 874-886.

65. Sloan EK, Priceman SJ, Cox BF, Yu S, Pimentel MA, Tangkanangnukul V et al. The sympathetic nervous system induces a metastatic switch in primary breast cancer. Cancer Res 2010; 70: 7042-7052.

66. Palm D, Lang K, Niggemann B, Drell TL, Masur K, Zaenker KS et al. The norepinephrinedriven metastasis development of PC-3 human prostate cancer cells in BALB/c nude mice is inhibited by $\beta$-blockers. Int J Cancer 2006; 118: 2744-2749.

67. Kao CJ, Martiniez A, Shi XB, Yang J, Evans CP, Dobi A et al. miR-30 as a tumor suppressor connects EGF/Src signal to ERG and EMT. Oncogene 2013.

68. Bockhorn J, Dalton R, Nwachukwu C, Huang S, Prat A, Yee K et al. MicroRNA-30c inhibits human breast tumour chemotherapy resistance by regulating TWF1 and IL-11. Nat Commun 2013; 4: 1393.

69. Yu F, Deng H, Yao H, Liu Q, Su F, Song E.. Mir-30 reduction maintains self-renewal and inhibits apoptosis in breast tumor-initiating cells. Oncogene 2010; 29: 4194-4204.

70. Zhang N, Wang X, Huo Q, Sun M, Cai C, Liu Z et al. MicroRNA-30a suppresses breast tumor growth and metastasis by targeting metadherin. Oncogene 2013; 33: 3119-3128.

Cell Death and Disease is an open-access journal published by Nature Publishing Group. This work is licensed under a Creative Commons Attribution 4.0 International License. The images or other third party material in this article are included in the article's Creative Commons license, unless indicated otherwise in the credit line; if the material is not included under the Creative Commons license, users will need to obtain permission from the license holder to reproduce the material. To view a copy of this license, visit http://creativecommons.org/licenses/by/4.0/

Supplementary Information accompanies this paper on Cell Death and Disease website (http://www.nature.com/cddis) 